\title{
Development of UPLC-Q-TOF-MS Coupled with Cation-exchange Solid-phase Extraction Method for the Determination of Ten Pyrrolizidine Alkaloids in Herbal Medicines
}

\author{
Zhimin Luo, Xuan LI, Lu Wang, Chun Chang, and Qiang Fu ${ }^{\dagger}$ \\ School of Pharmacy, Xi'an Jiaotong University, Xi'an 710061, China
}

\begin{abstract}
Pyrrolizidine alkaloids are secondary metabolites of plants and can cause significant hepatotoxicity in humans. In this study, a fast and simple method was developed to determine ten pyrrolizidine alkaloids (PAs) in six types of herbal medicines using ultra performance liquid chromatography-quadrupole-time of flight mass spectrometry (UPLC-Q-TOFMS). An efficient solid-phase extraction procedure was carried out by using strong cation-exchange cartridges and the parameters were optimized. The established analytical method was validated and the results showed that the method presented satisfactory accuracy and precision. The established method was successfully applied for the determination of PAs in six herbal plants, including Senecionis Scandentis Hebra, Arnebiae Radix, Asteris Radix Et Rhizoma, Farfarae Flos, Senecionis Cannabifolii Herba and Emilia sonchifolia. PAs were found in all of these herbal plant samples. Eight types of related commercial herbal drugs were also detected, six of them were detected with different amounts of PAs. This work not only provided a powerful technical platform for both qualitative and quantitative analysis of PAs in herbal medicines, but also obtained information concerning PAs in these herbal samples, which could provide reference to the government regulatory authorities and non-governmental organizations.
\end{abstract}

Keywords Pyrrolizidine alkaloids, herbal medicines, cation-exchange solid-phase extraction, UPLC-Q-TOF-MS

(Received June 24, 2019; Accepted July 30, 2019; Advance Publication Released Online by J-STAGE August 9, 2019)

\section{Introduction}

Herbal medicines were widely used to treat diseases and maintain human health for thousands of years in many countries. ${ }^{1}$ Over the past 20 years, herbal medicines have become increasingly popular not only in Asia, but also in Europe, North America and Australia. ${ }^{2}$ Patients and consumers commonly believe that herbal medicine and other herbal products are of natural origin and have less side effects. Along with the widespread use of herbal medicine, cases of herbal medicineinduced liver injury (HM-ILI) are frequently reported from many countries around the world. ${ }^{3,4}$ Researchers have found that the intrinsic types of HM-ILI are mostly caused by herbs with pyrrolizidine alkaloids (PAs)..$^{5-7}$

Pyrrolizidine alkaloids (PAs) are natural toxins and more than 660 PAs have been identified in over 6000 plants but especially in Boraginaceae, Asteraceae and Fabaceae families. ${ }^{8}$ PAs are a major cause of patient poisonings through the consumption of PA-containing medicinal plants, herbal tea, and herbal remedies. ${ }^{9,10}$ PAs can cause significant hepatomegalia, live venoocclusive disease, hepato-carcinogenicity, neurotoxicity, mutagenicity and embryotoxicity. ${ }^{11-13}$ Due to the toxic effects of PAs, a health-based guidance value was established, recommending that the daily intake of PAs should be less than $0.007 \mu \mathrm{g} \mathrm{kg}^{-1}$ body weight. ${ }^{14}$ However, many PAs-containing plants were still widely used as herbal medicine in many

† To whom correspondence should be addressed.

E-mail: fuqiang@mail.xjtu.edu.cn countries, especially in China, including Senecionis Scandentis Herba, Farfarae Flos, Arnebiae Radix, Asteris Radix Et Rhizoma, Senecionis Cannabifolii Herba and Emiliae Herba. ${ }^{15}$ For example, Farfarae Flos, as a popular folk medicine, is listed in many editions of Chinese Pharmacopoeia (ChP) and Korean Pharmacopoeia; it has been widely used to treat coughs, bronchitis and asthma in Asia. ${ }^{16,17}$ It has also been used as an herbal remedy for the same applications in Europe. ${ }^{18}$ Emilia sonchifolia, as a traditional medicinal plant, is widely used in most tropical and subtropical countries, such as China, India, Thailand, Nigeria and Brazil. ${ }^{19,20}$ In general, these six types of herbs were widely used as an individual drug or as a prescription together with other drugs for the antipyretic, antiphlogistic and antidotal purposes, in cases of measles, angina, urinary-tract infections, boils, pyoderma, mastitis, dysentery, cough, etc. .,5,15,18 $^{2,18}$ Because of lack of restrictions in most countries' pharmacopeias, the potential threats of PAs may be underestimated to human health. Therefore, it is urgent to monitor PAs in these medicinal plants in order to avoid risks to patients and consumers.

Due to the intoxication of PAs, many researchers have reported the detection of PAs in honey, ${ }^{14}$ tea, ${ }^{10}$ milk, ${ }^{21}$ salad, ${ }^{22}$ cereal ${ }^{23}$ and other food stuffs by using spectrophotometry, ${ }^{24}$ high-performance liquid chromatography (HPLC), ${ }^{8}$ micellar electrokinetic chromatography (MEKC), ${ }^{25}$ liquid chromatography-mass spectrometry (LC-MS), ${ }^{26}$ gas chromatography-mass spectrometry (GC-MS), ${ }^{27}$ etc. However, only few papers have reported the determination of PAs in herbal plants. Mroczek et al. established an HPLC method for the determination of senkirkine and senecionine in Symphytum sp., Petasites hybridus and Petasites albus, Tussilago farfara, Emilia coccinea and 


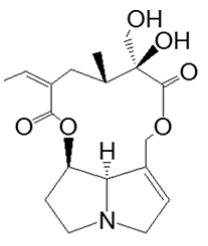

retrorsine

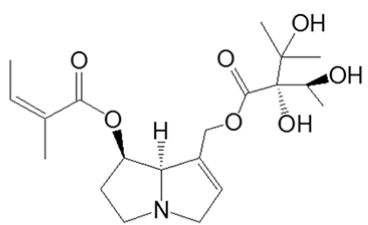

echimidine

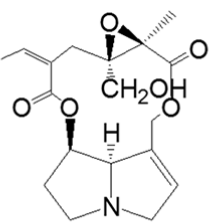

erucifoline

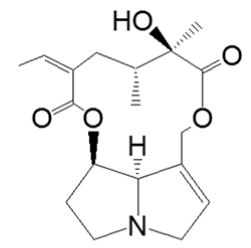

senecionine

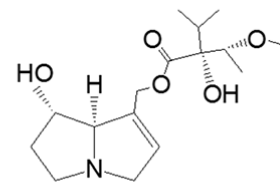

heliotrine

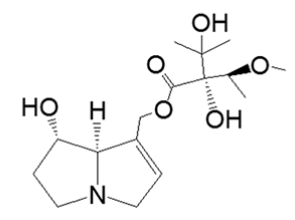

europine

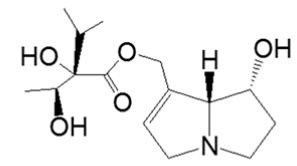

lycopsamine

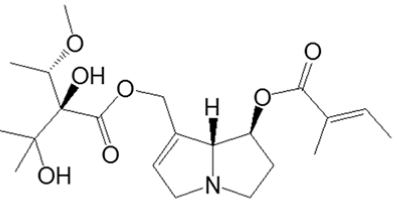

lasiocarpine

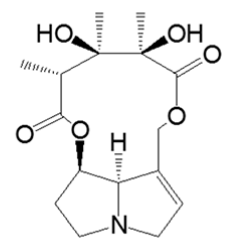

monocrotaline

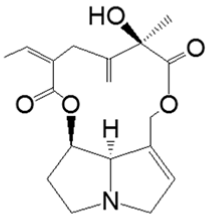

seneciphylline

Fig. 1 Chemical structure of hepatotoxic pyrrolizidine alkaloids.

Doronicum columnae, which were collected in Poland. ${ }^{8}$ Bodi et al. used the LC-MS method for the determination of PAs in peppermint, chamomile nettle, fennel, anise caraway and Melissa; the results showed that PAs were detected in nettle, fennel, anise caraway and melissa. ${ }^{22}$ Colegate et al. determined the dehydropyrrolizidine alkaloids (DPAs) in Amsinckia species and DPAs were detected in all specimens examined, ranging from 1 to $4000 \mu \mathrm{g} \mathrm{g}^{-1}$ of plant. ${ }^{28}$ As for Asteris Radix Et Rhizoma, Senecionis Cannabifolii Herba, Senecionis Scandentis Herba, Farfarae Flos, Arnebiae Radix, and Emiliae Herba, there are only a few papers that systematically determine PAs in them, to the best of our knowledge. Therefore, it is crucial to establish a highly sensitive and accurate analysis method for monitoring PAs in these six herbal medicines.

Up to now, liquid chromatography coupled to tandem mass spectrometry has become one of the fundamental techniques for the detection of PAs because of their high specificity and their low limit of detection. As an alternative to mass spectrometry instruments (MS), time-of-flight mass spectrometry (TOF-MS) shows high potential of high mass accuracy and mass resolution, which can complement other methods. ${ }^{27}$ It can generate high specificity, even though detecting lots of target compounds simultaneously. Compared with ESI-MS, TOF-MS possess the merits of more accurate mass determination in each scan, and a higher mass resolving power, which provide a greater degree of reduction in chemical noise, and thus the selectivity is also enhanced. ${ }^{29}$

With regard to the toxicity of PAs, the assessment of PAs intake, occurrence data in herbal medicine are required. Therefore, the aim of this study was to use ultra-performance liquid chromatography-quadrupole-time of flight mass spectrometry (UPLC-Q-TOF-MS) for the specific and accurate analysis of ten representative PAs in six types of herbal medicines, including Senecionis Scandentis Hebra, Arnebiae Radix, Asteris Radix Et Rhizoma, Farfarae Flos, Senecionis Cannabifolii Herba and Emilia sonchifolia. We aim to provide an effective tool for the qualification control and quantitative analysis of PAs in herbs and to provide information to guide the cautious use of these herbs for the pharmaceutical industry and market.

\section{Experimental}

\section{Reagents and chemicals}

Analytical standards (all with purity $\geq 95 \%$ ) of erucifoline, europine, retrorsine, seneciphylline, heliotrine, echimidine, lasiocarpine, senecionine and monocrotaline were purchased from ChemFaces (Wuhan, China). Lycopsamine was purchased from Toronto Research Chemicals (Toronto, Canada). The structures of ten PAs are shown in Fig. 1. Methanol (liquid chromatography grade) and acetonitrile (liquid chromatography grade) were purchased from Merck (Schwalbach, Germany). Formic acid (liquid chromatography grade, $\geq 98 \%$ ) was purchased from J\&K Chemical Ltd. (Beijing, China). Ammonium acetate $(99.99 \%)$ was provided by Shanghai Macklin Biochemical Co., Ltd. (Shanghai, China). Senecionis Scandentis Hebra, Arnebiae Radix, Asteris Radix Et Rhizoma, Farfarae Flos, Senecionis Cannabifolii Herba and Emilia sonchifolia were purchased from local herbal pharmacies and identified by Associate Prof. Xinxin Zhang. A scented tea sample was bought in a local supermarket. The commercial herbal drugs were purchased from a local Pharmacy, including pharyngitis tablets (Xi' an Keli Pharmaceutical Co., Ltd., China), anticough tablets (Teyi Pharmaceutical Group Co., Ltd., China), anti-cervical erosion suppository (Tonghua Wantong Pharmaceutical Co., Ltd., China), anticough granules for kids (Yunnan Baiyao Group Co., Ltd., China), anticough granules for adults (Jilin Yimintang Pharmaceutical Co., Ltd., China), anticough granules for adults (Renhe Pharmaceutical Co., Ltd., China), anti-acute bronchitis syrup (Chongqing Fuling Pharmaceutical Factory Co., Ltd., China) and slimming tablets (Kracie Holdings, Ltd., Japan). Ultrapure water was purified and filtered using a Milli-Q water-purification system (Bedford, 
USA). Dichloromethane and other chemicals were supplied by a local supplier. Solid-phase extraction columns with strong cation exchange cartridges (SCX-SPE) (Generik BCX, $500 \mathrm{mg}$, $3 \mathrm{~mL}$ ) were provided by Sepax Technologies, Inc. (Newark, USA). Millipore filters $(0.45 \mu \mathrm{m}, 17 \mathrm{~mm})$ were supplied by Nalgene (Rochester, USA).

\section{Apparatus}

A UPLC-Q-TOF-MS system (Waters Corporation, USA) with a chromatographic column UPLC HSS T3 $(1.0 \times 100 \mathrm{~mm}$, $1.8 \mu \mathrm{m}$ ) was used. A rotary evaporator RE-52 (Huanyu Equipment, China), benchtop centrifuges (Fisher Scientific, USA) and analytical weighing balance (Shimadzu AUX 220, Japan) were used.

\section{UPLC-Q-TOF-MS parameters}

In order to obtain a good chromatographic resolution and sensitivity, the UPLC conditions were optimized. Mobile phase A was $0.1 \mathrm{vol} \%$ formic acid in methanol, and mobile phase B was $0.2 \mathrm{mmol} \mathrm{L}^{-1}$ of ammonium acetate in water: The lineargradient program was set as follows: $0 \mathrm{~min}, 5 \mathrm{vol} \% \mathrm{~A} ; 4 \mathrm{~min}$, 95 vol\% A; 7 min, 95 vol\% A; 8 min, 5 vol\% A; 10 min, 5 vol\% A. The flow rate was $0.3 \mathrm{~mL} \mathrm{~min}^{-1}$, and the column temperature was maintained at $35^{\circ} \mathrm{C}$. One microliter of a sample was injected into the instrument. The instrument performed in the positive electrospray mode $\left(\mathrm{ESI}^{+}\right)$. For monitoring and confirmation analysis, the multiple-ion reaction monitoring mode was used. The source conditions were set as follows: source temperature, $100^{\circ} \mathrm{C}$; desolvation temperature, $500^{\circ} \mathrm{C}$; cone gas flow rate, $50 \mathrm{~L} \mathrm{~h}^{-1}$; desolvation gas flow rate, $800 \mathrm{~L} \mathrm{~h}^{-1}$. High-purity $\mathrm{N}_{2}$ was used as a desolvation and cone gas. Mass spectra were acquired over the range $m / z, 50-600$.

\section{Sample preparation}

Dried herbal plant samples were smashed by using a highspeed mixer and the obtained powder was sifted with a 30 mesh. Five grams of each sample were mixed and dissolved with $5 \mathrm{~mL}$ of 10 vol\% ammonium hydroxide for $1 \mathrm{~h}$. A $100-\mathrm{mL}$ volume of dichloromethane-methanol $(1: 1)$ was added, and then extracted by heating reflux for $3 \mathrm{~h}$. The extract was filtered and the residue was re-extracted three times. The extracted solutions were combined and evaporated. The residues were re-dissolved by $20 \mathrm{~mL}$ of methanol with $0.1 \mathrm{vol} \%$ formic acid. The obtained solutions were put into a refrigerator at $4{ }^{\circ} \mathrm{C}$ overnight, and then centrifuged for $15 \mathrm{~min}$ at $12000 \mathrm{rpm}$. The supernatants were kept at $4^{\circ} \mathrm{C}$ in a refrigerator for a SPE procedure.

Commercial herbal drug tablets and granules (5 g) were smashed by using mortar, and the obtained powder was sifted (30 mesh). The other extraction steps were the same as the herbal samples mentioned above. The commercial syrup $(5 \mathrm{~mL})$ and suppository $(5 \mathrm{~g})$ were directly mixed and dissolved with $5 \mathrm{~mL}$ of $10 \mathrm{vol} \%$ ammonium hydroxide for $1 \mathrm{~h}$. The other extraction steps were the same as the herbal samples mentioned above. The obtained supernatants were kept at $4^{\circ} \mathrm{C}$ in a refrigerator for the SPE procedure.

\section{SPE procedure}

Generik BCX columns were preconditioned with $6 \mathrm{~mL}$ of methanol and $6 \mathrm{~mL}$ of $0.05 \mathrm{M}$ sulfuric acid, respectively. The flow rates were maintained to $1 \mathrm{~mL} \mathrm{~min}^{-1}$ under a reduced pressure. Three milliliters of the obtained supernatant samples were loaded and then washed with methanol $(3 \mathrm{~mL})$. After that, $12 \mathrm{~mL}$ of methanol contained with $0-4 \mathrm{vol} \%$ of ammonium hydroxide was used to elute the alkaloids. The obtained solutions were evaporated by nitrogen gas. The dried residues were reconstituted with $1 \mathrm{~mL}$ of methanol and filtered through a $0.45-\mu \mathrm{m}$ Millipore filter. The obtained solutions were injected into the UPLC-Q-TOF system for analysis.

\section{Method validation}

The analytical method was validated following recommendations of the International Conference on Harmonization Q2(R1). ${ }^{30}$ The linearity of this method was valued through the preparation of six different calibration curves by spiking a tea sample, which had been detected without any of PAs, with different PAs mixed standard solutions before proceeding with the extraction. The final linear ranges of the analytes varied from 2 to $500 \mathrm{ng} \mathrm{mL}^{-1}$. The limit of detection (LOD) and the limit of quantitation (LOQ) were determined at signal-to-noise $(S / N)$ ratios of 3 and 10 , respectively. The precision and recovery of the method were evaluated by performing tests on three sets of spiked samples at three different concentrations $\left(5,10,50 \mathrm{ng} \mathrm{mL}^{-1}\right)$. The intra-day and inter-day repeatabilities were also estimated by analyzing spiked samples in a single day and for three days, respectively. The stability of the tested samples was tested with the spiked samples stored at room temperature $\left(25^{\circ} \mathrm{C}\right)$. The samples were analyzed in sextuplicate at $2,4,6,8,10 \mathrm{~h}$.

\section{Results and Discussion}

\section{Sample preparation}

Herbal samples usually contain a vast amount of different types of compounds; consequently, it is important to effectively reduce the matrix effects and purify targets through sample preparation. To minimize the action of matrix hydrophilic interfering compounds, ammonium hydroxide was used to transfer ionic alkaloids to free-state, and then a dichloromethanemethanol mixture was used to extract all PAs by heating reflux extraction.

\section{Solid phase extraction}

Although most of the hydrophilic interferences were removed after sample extraction, the huge amount of hydrophobic compounds was still presented and would interfere in the signal of targets. ${ }^{31}$ Further cleanup steps were necessary, and solidphase extraction was most widely used. ${ }^{32}$ Thereinto, strong cation exchange cartridges grafted with benzenesulfonic acid have been proved to be the most efficiency SPE columns for the purification of target PAs, and to remove the interferences of all samples. ${ }^{33}$ However, an efficient release of targets from the cartridges to achieve a high recovery was usually hard.

For the SPE procedure, the loaded solutions were with $0.1 \mathrm{vol} \%$ formic acid to generate the protonated amine group of PAs, which was a benefit for the ionic interaction between the tertiary amine group of PAs and the sulfonic anion group of the SCX sorbent. The elution of PAs relied on the SCX-SPE procedure in term of the composition and $\mathrm{pH}$ of the elution solvent. The extract of tea samples which had been previously detected without any PAs was used as the model plant sample, and added with known amounts of a ten PA standards mixture. During sample loading, the positively charged PAs strongly interacted with the sulfonic anion groups of the SCX sorbent. Then, the elution solvents (ammonium hydroxide from $0-4 \mathrm{vol} \%$ in methanol) were used to cleave the ionic interaction between PAs and the SCX sorbent. The elution efficiency values of different elution solvents are shown in Fig. 2.

Because of the strong interaction between PAs and the SCX sorbent, the elution solvent of methanol without ammonium 
hydroxide showed lower elution efficiency. Along with the amount of ammonium hydroxide increased in the elution solvent, the ionic interaction between PAs and the SCX sorbent was gradually broken. The elution solvents of methanol with 3 and 4 vol\% ammonium hydroxide exhibited the similar elution ability. It was reported that PAs showed a degradation possibility under a high concentration of ammonium hydroxide. ${ }^{34}$ Therefore, 3 vol\% ammonium hydroxide in methanol was selected as the final optimized elution solvent. Figure 3 shows the total ion chromatograms of PAs extracted from an Emilia sonchifolia sample before and after being treated by SPE. There were seven PAs detected in the Emilia sonchifolia sample, including echimidine, erucifoline, europine, lycopsamine, retrorsine, senecionine and seneciphylline. After cationexchange solid-phase extraction, the interference of matrices was efficiently eliminated and the targeted PAs were well enriched.

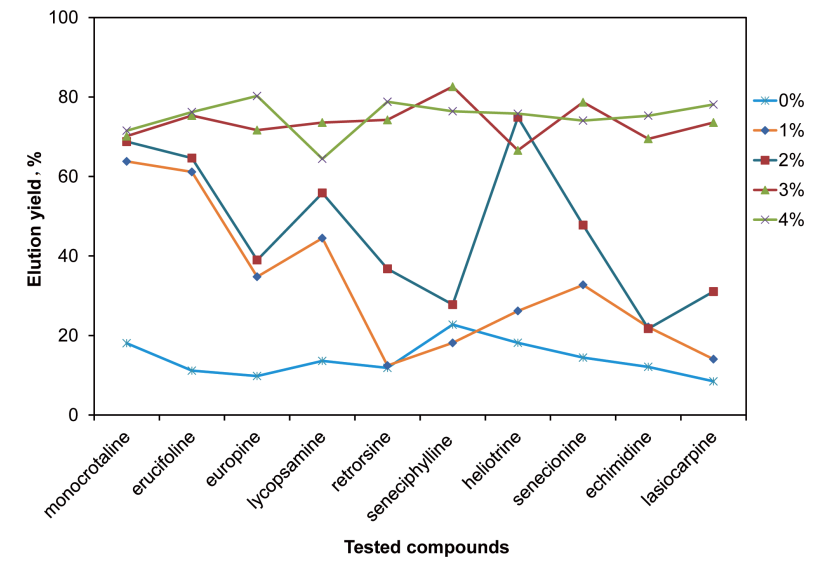

Fig. 2 Elution yields of PAs under different percentages of ammonium hydroxide in methanol.
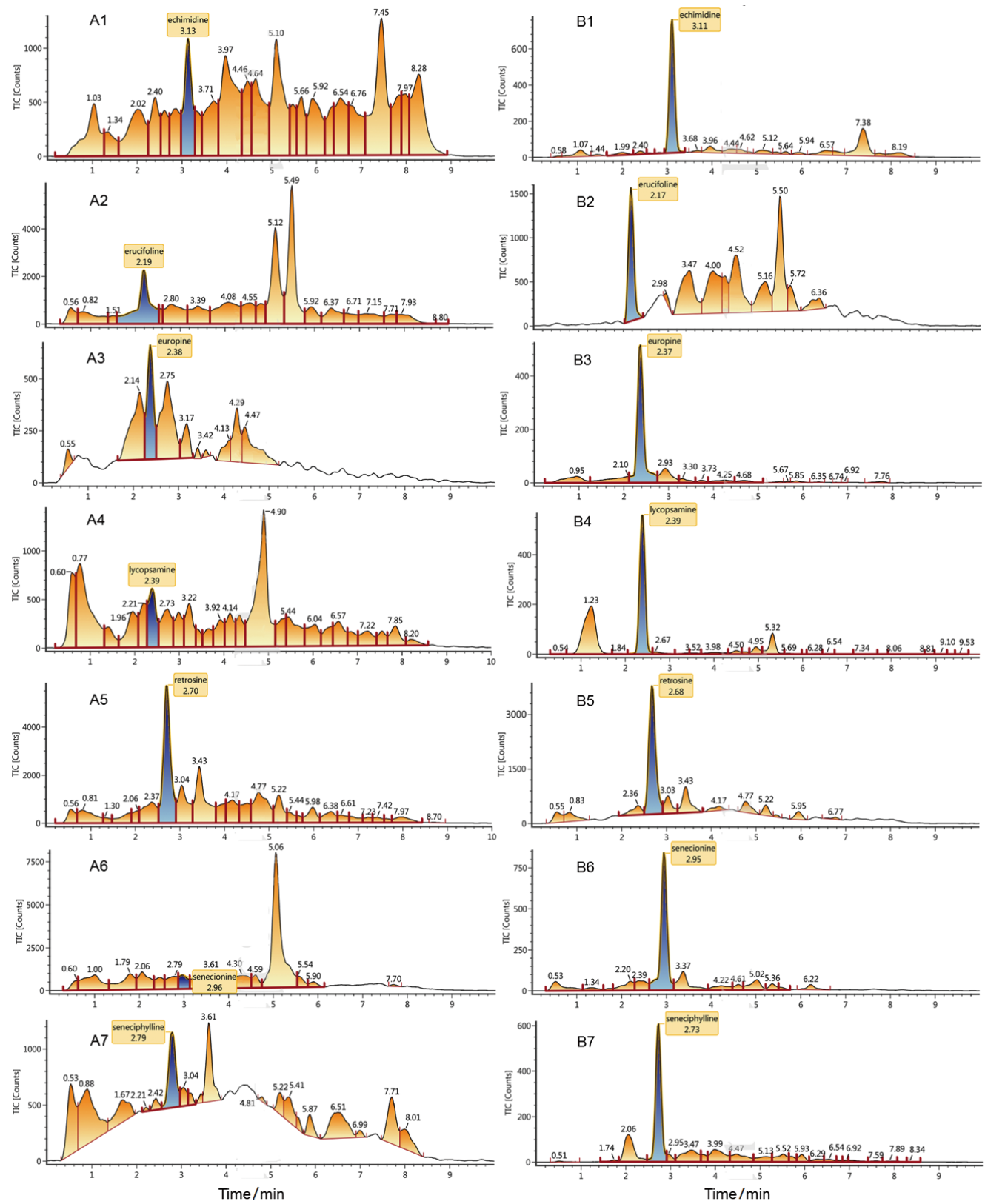

Fig. 3 Total ion chromatograms of PAs extracted from Emilia sonchifolia samples before (A1 - A7) and after (B1 - B7) SPE treated. 


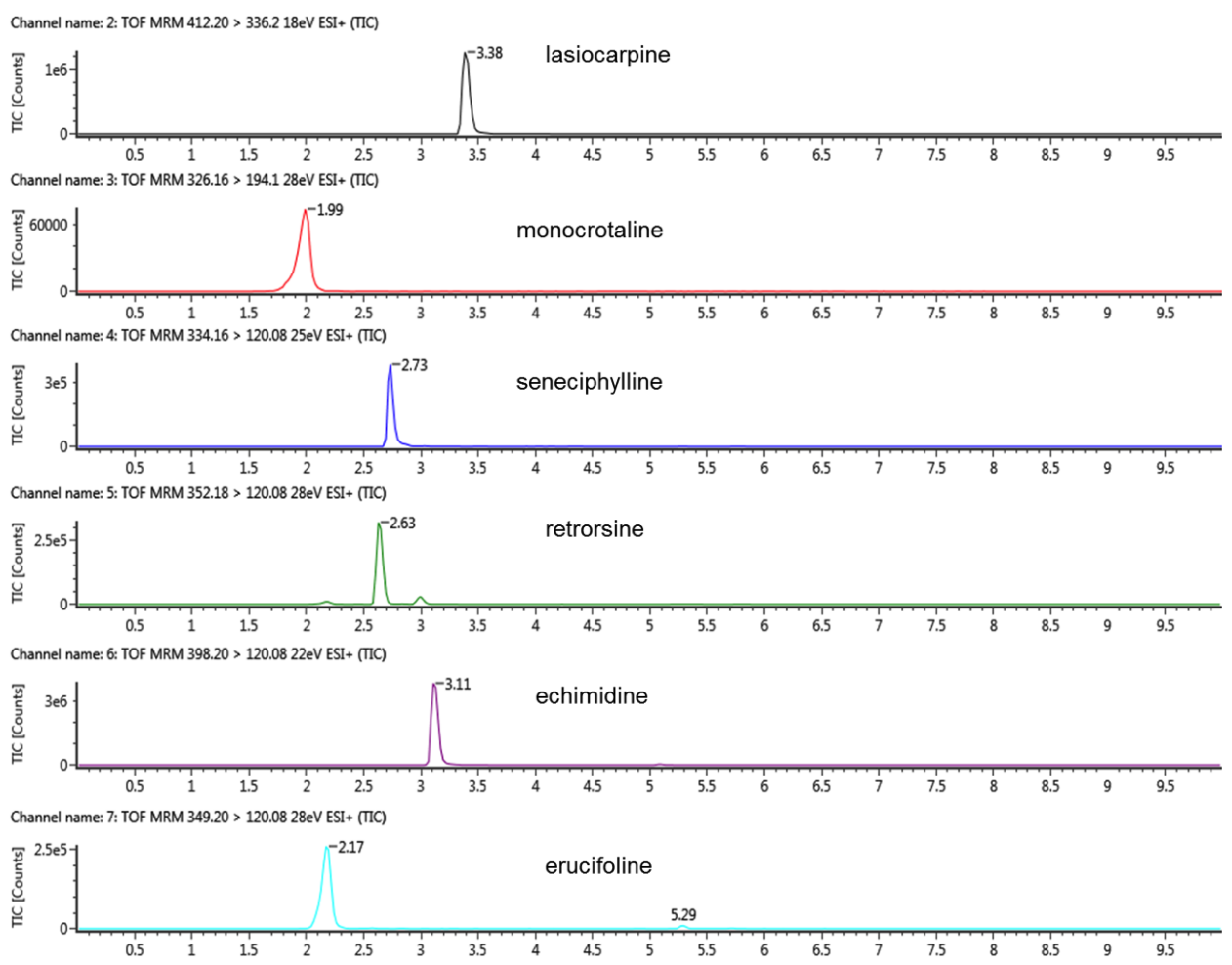

Channel name: 8: TOF MRM 314.20 > 138.1 20eV ESI+ (TIC)

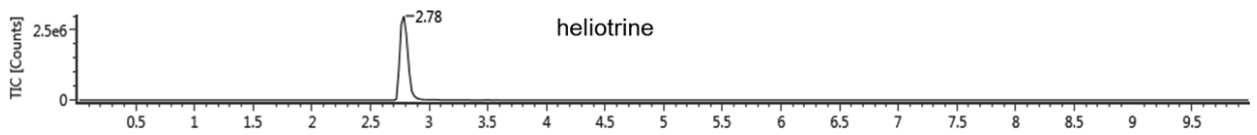

Channel name: 9: TOF MRM 330.20 > 138.1 22eV ESI+ (TIC)

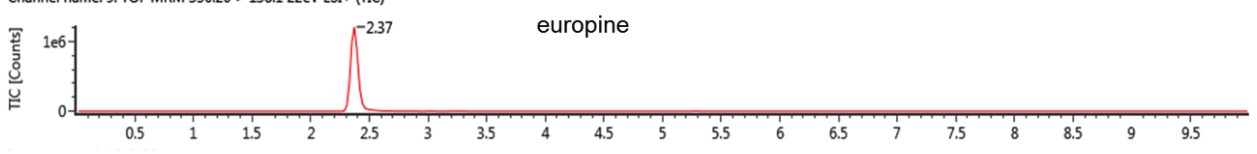

Channel name: 10: TOF MRM 300.18 > 94.06 28eV ESI+ (TIC)

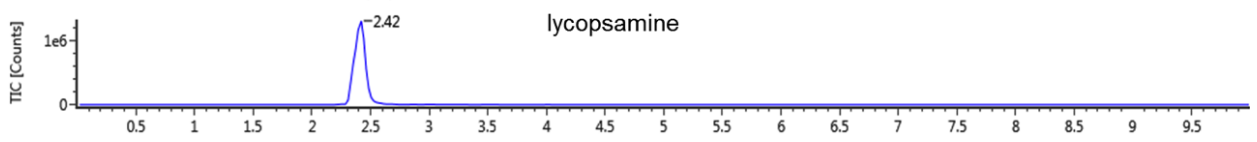

Channel name: 13: TOF MRM $336.20>138.228 \mathrm{eV} \mathrm{ESI+} \mathrm{(TC)}$

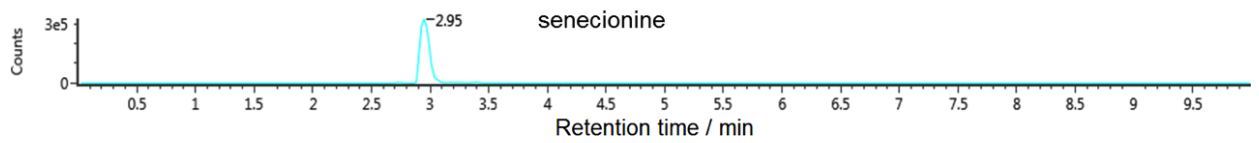

Fig. 4 Topical chromatograms of PAs obtained from spiked model samples after SPE treatment.

\section{Method development}

The UPLC conditions, including type of column, injection volume, mobile phase, and flow rate, were investigated to achieve the best performance related to the peak symmetry, chromatographic resolution and analysis time. In this study, three columns were evaluated including Sepax C18 $(2.1 \times 100$ $\mathrm{mm}, 3 \mu \mathrm{m})$, Syncronis C18 $(2.1 \times 100 \mathrm{~mm}, 1.7 \mu \mathrm{m})$ and Waters UPLC HSS T3 $(1.0 \times 100 \mathrm{~mm}, 1.8 \mu \mathrm{m})$. Ultimately, the Waters UPLC HSS T3 column was applied because of the better separating effect. Different gradients and solvents (methanol and acetonitrile, aqueous ammonium acetate and formic acid of different $\mathrm{pH}$ values) were checked. The results showed that $0.1 \mathrm{vol} \%$ of a formic acid aqueous solution with methanol using the gradient mode showed a higher response in the $\mathrm{ESI}^{+}$mode. A low proportion of methanol ( $5 \mathrm{vol} \%)$ during the first minutes of the run was used to elute the hydrophilic PAs such as monocrotaline. During the end of the gradient, $95 \mathrm{vol} \%$ of mobile phase A could provide satisfactory column cleaning, thereby minimizing the carry-over effects. At the end, a 3-min re-equilibration step with 5 vol\% of mobile phase A was used to maintain stable and reproducible separation, and to prolong the column lifetime. ${ }^{35}$ A topical chromatogram of PAs obtained from the UPLC-Q-TOF-MS of an extract of a spiked model sample after being treated by SPE is shown in Fig. 4 .

In order to obtain the maximum sensitivity and the highest possible resolution, the spiked tea samples were injected into UPLC-Q-TOF-MS and the operation conditions were optimized, including the collision energies, capillary and cone voltages, desolvation temperature and desolvation and cone gas. The results are given in Table 1 . PAs were quantitatively determined in the MRM acquisition mode. The most intense fragment of each PAs was used as the quantification ion, and another transition as the confirmation ion. 
Table 1 Q-TOF-MS parameters of PA

\begin{tabular}{|c|c|c|c|c|c|}
\hline Compound & $\begin{array}{l}\text { Precursor } \\
\text { ion } m / z\end{array}$ & $\begin{array}{l}\text { Product } \\
\text { ion } m / z\end{array}$ & $\begin{array}{c}\text { Cone } \\
\text { voltage/V }\end{array}$ & $\begin{array}{c}\text { Capillary } \\
\text { energy/kV }\end{array}$ & $\begin{array}{c}\text { Retention } \\
\text { time/min }\end{array}$ \\
\hline Seneciphylline & 334.16 & $\begin{array}{l}120.08 \\
138.09\end{array}$ & 25 & 2.0 & 2.73 \\
\hline Lasiocarpine & 412.20 & $\begin{array}{l}336.20 \\
120.05\end{array}$ & 18 & 1.8 & 3.38 \\
\hline Retrorsine & 352.18 & $\begin{array}{l}120.08 \\
276.15\end{array}$ & 28 & 2.0 & 2.63 \\
\hline Heliotrine & 314.20 & $\begin{array}{l}138.10 \\
156.10\end{array}$ & 20 & 1.8 & 2.78 \\
\hline Echimidine & 398.20 & $\begin{array}{l}120.08 \\
220.09\end{array}$ & 22 & 1.8 & 3.11 \\
\hline Monocrotaline & 326.16 & $\begin{array}{l}194.10 \\
280.01\end{array}$ & 28 & 2.0 & 1.99 \\
\hline Erucifoline & 349.20 & $\begin{array}{l}120.08 \\
267.00\end{array}$ & 28 & 2.1 & 2.17 \\
\hline Europine & 330.20 & $\begin{array}{l}138.10 \\
120.10\end{array}$ & 22 & 1.8 & 2.37 \\
\hline Lycopsamine & 300.18 & $\begin{array}{l}94.06 \\
156.1\end{array}$ & 28 & 1.8 & 2.42 \\
\hline Senecionine & 336.20 & $\begin{array}{l}120.10 \\
138.20\end{array}$ & 28 & 2.0 & 2.95 \\
\hline
\end{tabular}

\section{Method validation}

Along with the increasing numbers of detected ions, the sensitivity of LC-MS was likely to decrease. ${ }^{36}$ In addition, the inherent instability of the ESI-MS response made the confident quantitation of the analytes unreliable. ${ }^{14}$ However, it would not occur in the LC-Q-TOF-MS assay, since the TOF analyzer not only can qualitatively analyze the unknown compounds, but also own the merits of high sensitivity for a full-spectrum scan. ${ }^{37}$ We conducted quantitative assays of ten PAs with authentic standards in six herbal plants. Quantification of the analytes was completed using linear regression and external calibration. Due to the difference in the concentration between different samples, a wide linear range was used from 2 to $500 \mathrm{ng} \mathrm{mL}^{-1}$. Calibration curves of the herbal plants were analyzed for each PAs component by a linear-regression analysis. All of the calibration curves were linear upon a visual inspection. Table 2 shows the regression equation and the linear regression coefficients for the PAs; all curves presented correlation coefficient $\left(r^{2}\right)$ values greater than 0.999, indicating excellent linearity.

As shown in Table 3, the LOD and LOQ values obtained in this study, respectively, ranged from 0.05 to $0.54 \mathrm{ng} \mathrm{mL}^{-1}$ and from 0.15 to $1.77 \mathrm{ng} \mathrm{mL}^{-1}$, indicating high sensitivity for the determination of trace PAs in these herbal plants. Because the test materials contained different classes of PAs, the detected types of PAs were varied in the test materials. As an example, europine and lycopsamine were detected in Arnebiae Radix, but the other analytes of interest were not detected; so accuracy and precision data were collected only for the PAs detected. The results showed that the accuracies of all of the analytes at three levels ranged from 75.1 to $110.1 \%$, and the relative standard deviations (RSDs) were less than $11.8 \%$ in those spiked samples, indicating that the established analytical method is suitable and reliable for the determination of the PAs in six herbal samples. The sample stability test was carried out at 2, 4, 6, 8, $10 \mathrm{~h}$. During the period, the solutions were stored at room temperature $\left(25^{\circ} \mathrm{C}\right)$. As shown in Table 4 , the recovery rate of ten tested compounds showed relatively good stability during the test time. The results demonstrated that the method is stable and reliable for the determination of PAs in herbal samples.
Table 2 Calibration curve parameters for plant samples obtained in the external standard calibration curve in the range $2-500 \mathrm{ng} \mathrm{mL}^{-1}$

\begin{tabular}{lll}
\hline \multicolumn{1}{c}{ Compound } & Regression equation & $r^{2}$ \\
\hline Erucifoline & $y=574.56 x+162.87$ & 0.9992 \\
Retrorsine & $y=623.85 x-1334.1$ & 0.9997 \\
Echimidine & $y=7000.4 x+7198.9$ & 0.9993 \\
Heliotrine & $y=6031.1 x-400.55$ & 0.9999 \\
Europine & $y=2744.4 x-1085.3$ & 0.9999 \\
Seneciphylline & $y=1390.6 x+2545.2$ & 0.9999 \\
Lasiocarpine & $y=1718.8 x+1628.3$ & 0.9994 \\
Monocrotaline & $y=270.94 x-2289.3$ & 0.9991 \\
Lycopsamine & $y=5986 x-6445.8$ & 0.9999 \\
Senecionine & $y=2248.1 x+551.69$ & 0.9990 \\
\hline
\end{tabular}

\section{PAs in herbal plant samples}

With the extensive use of herbal medicines, the safety of herbal medicines was also of wide concerned. For example, herbal medicines that contained nephrotoxic aristolochic acids have been strictly limited in many countries. ${ }^{38}$ However, the hepatotoxic PAs were still neglected by many government regulatory authorities. In this study, we investigated ten representative PAs in six herbal medicines. The results are shown in Fig. 5.

The first exposure of hepatotoxicity of PAs was due to the consumption of Senecio spp. contaminated flour in South Africa in $1920 .{ }^{39}$ Since then, people noticed that the presence of PAs could result in liver damage and then, subsequently, hepatic veno-occlusive disease (HVOD). PAs poisoning incidents have also been continuously reported. ${ }^{7,39,40}$ However, due to the complicated pathogenesis of HVOD, only a few cases of PA-induced HVOD were confirmed and reported. ${ }^{41}$ The latest reported case of PA-induced HVOD in China was a 23-year old woman in $2017 .{ }^{42}$ She had taken Runyirong capsules in which $S$. scandens was contained. In addition, because of a slow presentation of toxic symptoms, the toxic effects are often diagnosed when it is too late. Here, seven PAs, including erucifoline, europine, lycopsamine, retrorsine, seneciphylline, echimidine, lasiocarpine and senecionine, were detected in Senecionis Scandentis Hebra, and the total amount of PAs reached up to $5.6 \mu \mathrm{g} \mathrm{g}^{-1}$. Thereinto, the concentration of senecionine was $0.13 \mu \mathrm{g} \mathrm{g}^{-1}$. This result is consistent with a previous report that summarized 0.1 to $10 \mu \mathrm{g} \mathrm{g}^{-1}$ of the concentration of senecionine in Senecio spp. ${ }^{2}$ Considering the hepatotoxicity of PAs, ChP 2015 has limited the concentration of adonifoline to less than $0.004 \% .{ }^{15}$ However, in view of the multicomponents and high concentrations of PAs in Senecionis Scandentis Hebra, we strongly suggest that the Pharmacopeia could improve the supervision for other types of PAs.

The herbal preparations consisting of Senecionis Cannabifolii Herba, such as Fanhuncao granules or Feining oral liquids, were frequently used to cure viral influenza, pneumonia, and bronchitis in China. ${ }^{15}$ However, Senecionis Cannabifolii Herba also belongs to the genus of Senecio, and few reports studied the content of PAs in it as far as we know. In this study, five PAs were detected in Senecionis Cannabifolii Herba and the total content was $1.33 \mu \mathrm{g} \mathrm{g}^{-1}$, indicating the potential hepatotoxicity of these herbal preparations. Unfortunately, PAs of Senecionis Cannabifolii Herba were not limited in ChP.

It is recorded that Emilia sonchifolia, as the main raw material of Huahong Tablet, is mainly used to treat gynecological inflammation, including irregular menstruation, dysmenorrhea, endometritis, adnexitis and pelvic inflammation. ${ }^{15}$ However, 
Table 3 Recovery and precision results summary for PAs containing plants

\begin{tabular}{|c|c|c|c|c|c|c|c|c|c|c|c|c|}
\hline \multirow{2}{*}{ Test material } & \multirow{2}{*}{ Analyte } & \multirow{2}{*}{$\begin{array}{l}\text { LOD/ } \\
\text { ng mL } \mathrm{mL}^{-1}\end{array}$} & \multirow{2}{*}{$\begin{array}{c}\mathrm{LOQ} / \\
\mathrm{ng} \mathrm{mL}^{-1}\end{array}$} & \multicolumn{3}{|c|}{$\begin{array}{c}\text { Accuracy } \\
\text { Mean, } \%(n=6)\end{array}$} & \multicolumn{3}{|c|}{$\begin{array}{l}\text { Intra-day variability } \\
\quad \text { RSD, } \%(n=6)\end{array}$} & \multicolumn{3}{|c|}{$\begin{array}{l}\text { Inter-day variability } \\
\text { RSD, } \%(n=6)\end{array}$} \\
\hline & & & & 5 & 10 & 50 & 5 & 10 & 50 & 5 & 10 & 50 \\
\hline \multirow[t]{7}{*}{ Emilia sonchifolia } & Europine & 0.10 & 0.32 & 84.7 & 93.5 & 100.1 & 4.1 & 3.3 & 9.2 & 5.9 & 3.8 & 4.4 \\
\hline & Lycopsamine & 0.20 & 0.61 & 76.9 & 84.7 & 96.4 & 5.6 & 4.5 & 2.8 & 7.7 & 2.7 & 3.7 \\
\hline & Retrorsine & 0.28 & 0.92 & 82.8 & 92.5 & 98.3 & 7.2 & 3.5 & 5.6 & 5.9 & 4.2 & 4.3 \\
\hline & Seneciphylline & 0.16 & 0.55 & 75.4 & 76.3 & 89.6 & 2.3 & 7.6 & 3.1 & 7.2 & 1.9 & 2.9 \\
\hline & Echimidine & 0.20 & 0.70 & 86.7 & 79.5 & 83.5 & 5.7 & 9.1 & 3.9 & 7.1 & 5.8 & 6.5 \\
\hline & Lasiocarpine & 0.14 & 0.48 & 91.1 & 90.6 & 105.4 & 2.5 & 5.2 & 7.4 & 6.0 & 3.1 & 5.3 \\
\hline & Senecionine & 0.12 & 0.40 & 95.9 & 91.2 & 93.5 & 4.8 & 2.8 & 3.8 & 4.9 & 4.6 & 4.1 \\
\hline \multirow{4}{*}{$\begin{array}{l}\text { Asteris Radix Et } \\
\text { Rhizoma }\end{array}$} & Echimidine & 0.12 & 0.38 & 83.1 & 89.3 & 94.9 & 3.1 & 9.2 & 4.8 & 1.2 & 1.5 & 2.8 \\
\hline & Erucifoline & 0.54 & 1.77 & 94.0 & 88.7 & 103.7 & 3.9 & 8.6 & 3.6 & 4.9 & 3.5 & 5.8 \\
\hline & Retrorsine & 0.17 & 0.55 & 91.8 & 93.6 & 101.5 & 1.8 & 3.6 & 1.5 & 4.1 & 4.2 & 5.4 \\
\hline & Seneciphylline & 0.11 & 0.37 & 83.2 & 85.9 & 88.1 & 3.1 & 5.9 & 8.0 & 1.8 & 1.0 & 1.8 \\
\hline \multirow[t]{2}{*}{ Arnebiae Radix } & Europine & 0.27 & 0.89 & 76.0 & 96.7 & 75.3 & 3.9 & 6.6 & 5.2 & 3.8 & 7.6 & 7.5 \\
\hline & Lycopsamine & 0.30 & 1.00 & 81.1 & 104.0 & 110.1 & 8.5 & 4.0 & 10.1 & 6.3 & 4.9 & 2.1 \\
\hline Senecionis & Erucifoline & 0.47 & 1.55 & 87.9 & 91.9 & 102.6 & 7.9 & 1.8 & 2.5 & 5.0 & 6.9 & 7.4 \\
\hline \multirow[t]{6}{*}{ Scandentis Hebra } & Europine & 0.21 & 0.68 & 75.1 & 83.9 & 87.2 & 5.0 & 3.9 & 7.2 & 10.7 & 4.2 & 3.2 \\
\hline & Lycopsamine & 0.19 & 0.64 & 82.2 & 89.9 & 87.0 & 7.4 & 8.7 & 7.0 & 11.7 & 1.8 & 2.0 \\
\hline & Retrorsine & 0.08 & 0.26 & 103.1 & 81.9 & 104.2 & 3.0 & 1.9 & 4.2 & 5.4 & 2.6 & 3.9 \\
\hline & Seneciphylline & 0.11 & 0.36 & 88.3 & 85.2 & 96.0 & 8.3 & 5.2 & 5.9 & 4.6 & 3.8 & 2.5 \\
\hline & Echimidine & 0.10 & 0.34 & 100.3 & 97.6 & 98.2 & 9.5 & 7.5 & 8.2 & 5.3 & 10.7 & 2.2 \\
\hline & Senecionine & 0.27 & 0.90 & 91.0 & 96.5 & 103.5 & 3.9 & 6.4 & 3.5 & 2.3 & 2.6 & 3.0 \\
\hline \multirow[t]{6}{*}{ Farfarae Flos } & Erucifoline & 0.50 & 1.65 & 88.7 & 89.3 & 103.1 & 8.6 & 6.7 & 3.0 & 4.9 & 1.6 & 4.7 \\
\hline & Europine & 0.11 & 0.36 & 83.7 & 93.1 & 93.9 & 3.7 & 3.0 & 3.9 & 2.4 & 2.9 & 4.0 \\
\hline & Lycopsamine & 0.19 & 0.64 & 89.8 & 79.2 & 101.7 & 9.7 & 9.1 & 3.7 & 4.5 & 1.5 & 3.3 \\
\hline & Heliotrine & 0.08 & 0.26 & 77.0 & 77.5 & 100.2 & 3.9 & 3.4 & 3.2 & 11.8 & 9.6 & 3.1 \\
\hline & Echimidine & 0.12 & 0.40 & 79.3 & 78.6 & 81.7 & 7.6 & 6.4 & 2.6 & 2.7 & 4.0 & 1.6 \\
\hline & Senecionine & 0.05 & 0.15 & 76.5 & 79.1 & 87.2 & 7.5 & 6.8 & 4.5 & 5.8 & 2.7 & 2.5 \\
\hline Senecionis & Erucifoline & 0.48 & 1.59 & 84.9 & 93.3 & 105.0 & 2.3 & 2.2 & 4.3 & 3.6 & 2.5 & 3.8 \\
\hline Cannabifolii & Heliotrine & 0.11 & 0.35 & 82.8 & 106.7 & 106.4 & 2.7 & 3.9 & 3.2 & 7.9 & 4.3 & 6.3 \\
\hline \multirow[t]{3}{*}{ Herba } & Echimidine & 0.07 & 0.23 & 80.8 & 89.4 & 102.3 & 6.4 & 2.4 & 4.9 & 3.6 & 2.2 & 4.3 \\
\hline & Lasiocarpine & 0.06 & 0.21 & 95.1 & 95.0 & 109.8 & 5.0 & 3.3 & 4.9 & 2.6 & 2.9 & 3.6 \\
\hline & Monocrotaline & 0.47 & 1.57 & 78.1 & 79.9 & 89.3 & 5.4 & 6.7 & 3.3 & 9.8 & 8.3 & 3.5 \\
\hline
\end{tabular}

Table 4 Stability of tested compounds $(n=6)$

\begin{tabular}{|c|c|c|c|c|c|c|c|c|c|c|}
\hline \multirow{2}{*}{ Compound } & \multicolumn{2}{|l|}{$2 \mathrm{~h}$} & \multicolumn{2}{|l|}{$4 \mathrm{~h}$} & \multicolumn{2}{|l|}{$6 \mathrm{~h}$} & \multicolumn{2}{|l|}{$8 \mathrm{~h}$} & \multicolumn{2}{|c|}{$10 \mathrm{~h}$} \\
\hline & Recovery, \% & RSD, $\%$ & Recovery, \% & $\mathrm{RSD}, \%$ & Recovery, \% & $\mathrm{RSD}, \%$ & Recovery, $\%$ & $\mathrm{RSD}, \%$ & Recovery, \% & RSD, \% \\
\hline Lycopsamine & 100.6 & 2.9 & 99.2 & 4.5 & 103.0 & 3.6 & 101.0 & 1.1 & 99.7 & 2.5 \\
\hline Seneciphylline & 99.6 & 5.4 & 98.6 & 4.0 & 99.7 & 3.0 & 101.6 & 4.3 & 99.0 & 4.8 \\
\hline Monocrotaline & 99.9 & 4.4 & 101.8 & 5.2 & 98.7 & 3.3 & 100.6 & 7.1 & 98.3 & 5.4 \\
\hline Retrorsine & 100.3 & 4.5 & 101.5 & 3.5 & 100.2 & 3.8 & 99.6 & 3.2 & 100.5 & 5.1 \\
\hline Senecionine & 102.3 & 3.6 & 102.3 & 4.5 & 103.1 & 4.2 & 98.5 & 5.7 & 99.5 & 3.5 \\
\hline Heliotrine & 99.0 & 5.8 & 98.6 & 4.8 & 100.8 & 1.6 & 95.8 & 5.8 & 99.1 & 5.5 \\
\hline Lasiocarpine & 98.9 & 3.9 & 99.5 & 2.1 & 97.8 & 0.9 & 99.1 & 5.6 & 98.1 & 5.6 \\
\hline Erucifoline & 98.1 & 3.4 & 98.5 & 4.2 & 99.0 & 5.1 & 99.3 & 0.8 & 98.6 & 5.7 \\
\hline Echimidine & 99.7 & 3.6 & 98.5 & 5.7 & 100.3 & 2.8 & 98.6 & 3.7 & 101.2 & 4.9 \\
\hline Europine & 99.4 & 5.3 & 96.3 & 3.7 & 103.4 & 4.5 & 99.9 & 3.1 & 98.3 & 4.5 \\
\hline
\end{tabular}

seven types of PAs were detected in Emilia sonchifolia with a total amount of $4.35 \mu \mathrm{g} \mathrm{g}^{-1}$. One pill of Huahong tablet contains $5 \mathrm{~g}$ of Emilia sonchifolia, which means it may contain around $22 \mu \mathrm{g}$ of PAs per pill. The result suggests that the use of Huahong tablet may cause intoxication.

Arnebiae Radix is one of the most commonly used herbal medicines in many countries for the treatment of inflammation and wounds. At least 15 types of preparations in $\mathrm{ChP}$ consist of Arnebiae Radix. ${ }^{15}$ It was reported that PAs were detected in Arnebiae Radix, such as europine, heliotrine, lycopsamine, echimidine and intermedine. ${ }^{43}$ In this study, only europine and lycopsamine were detected in Arnebiae Radix. The total content was $0.09 \mu \mathrm{g} \mathrm{g}^{-1}$, which was lower than other reports. The possible reason was that the types and concentrations of PAs in Arnebiae Radix would be varied in different species and different areas. ${ }^{41}$

Asteris Radix Et Rhizoma is used as the monarch drug in many prescriptions, such as Zibai Zhisou Capsule and Ziwan Zhike electuary, and these prescriptions are widely used to treat cough and mycoplasma pneumonia, especially for children. ${ }^{44}$ However, erucifoline, retrorsine, seneciphylline and echimidine were detected and the total content was $1.84 \mu \mathrm{g} \mathrm{g}^{-1}$ in Asteris Radix Et Rhizoma, suggesting the potential hepatotoxicity of these prescriptions. 

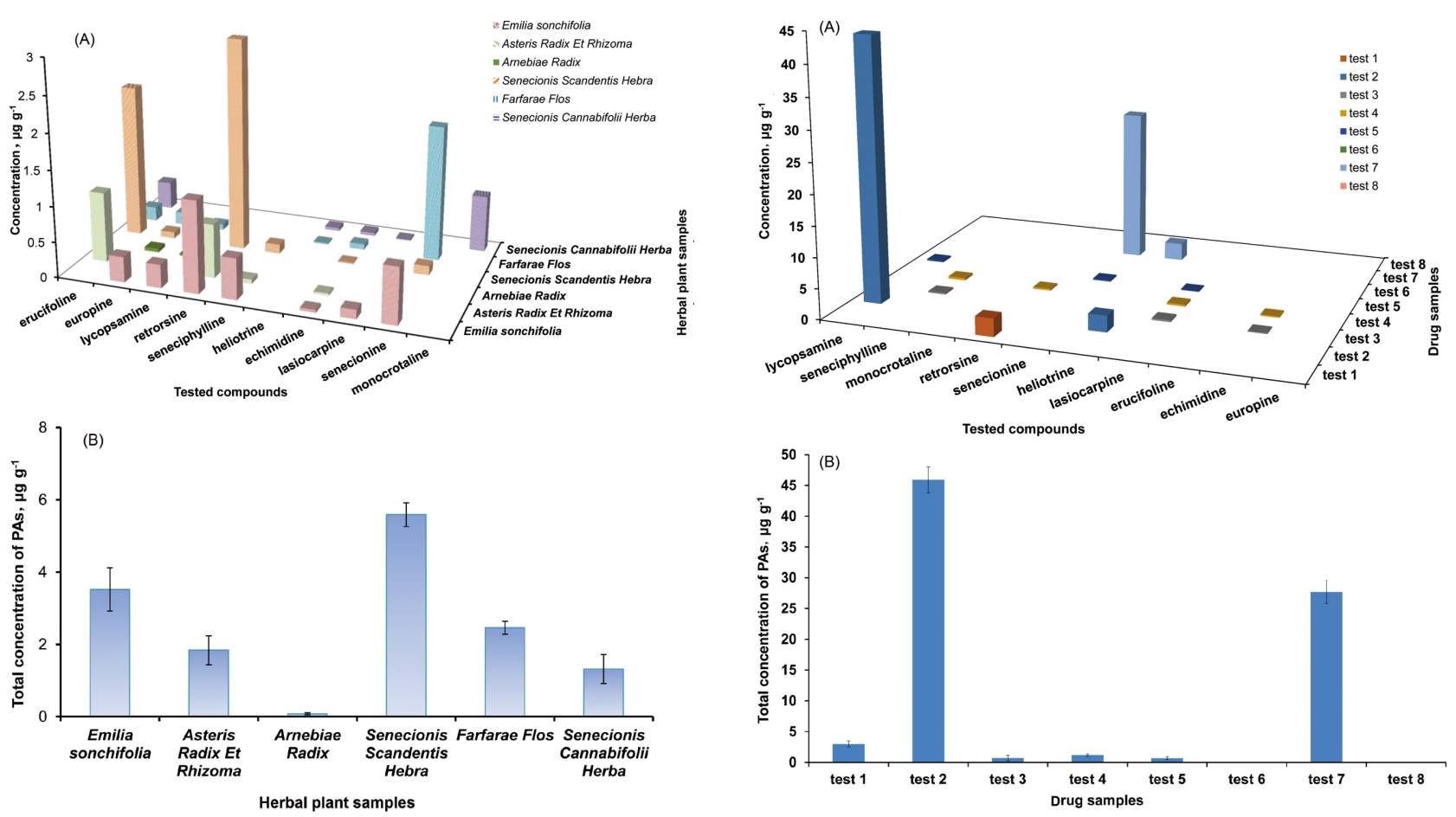

Fig. 5 Concentrations of PAs in herbal plant samples. Concentration of the single analytes in herbal plant samples; B, total concentration of PAs in herbal plant samples.

Most researchers mainly focused on Farfarae Flos's active compounds, such as tussilagone, ${ }^{17,45}$ but only few reported on the hepatotoxicity of Farfarae Flos. ${ }^{18}$ Our results showed that six types of PAs were detected and the total content reached to $2.46 \mu \mathrm{g} \mathrm{g}^{-1}$ in Farfarae Flos. Consequently, the potential hepatotoxicity of Farfarae Flos should be highly stressed.

In light of the guidance of ChP, ten grams of these herbal plants were usually used in each dose, suggesting that the intake of PAs in each type of these herbs tended to over the limitation of $0.007 \mu \mathrm{g} \mathrm{kg}^{-1}$ body weight. Consequently, these six herbal plants showed high potential for hepatotoxicity.

\section{PAs in commercial herbal drug samples}

Eight types of commercial herbal drug samples that contained Senecionis Scandentis Hebra, Arnebiae Radix, Asteris Radix Et Rhizoma, Farfarae Flos, Senecionis Cannabifolii Herba, Emilia sonchifolia or peppermint were also determined by using the established method. The results are shown in Fig. 6. The pharyngitis tablet samples were detected with $2.97 \mu \mathrm{g} \mathrm{g}^{-1}$ of PAs, which might be attributed to the constituent of Farfarae Flos in the pharyngitis tablet. The anticough tablet samples were detected with high concentrations of PAs $\left(45.92 \mu \mathrm{g} \mathrm{g}^{-1}\right)$. A possible reason was that the extract of Asteris Radix Et Rhizoma was one of the main constituents in the anticough tablet. This type of anticough tablet showed high threats to human health. Two types of anticough granules were detected with certain amount of PAs, which could be ascribed to the main constituents of Senecionis Cannabifolii Herba in them. Anticough granules for kids were also detected with $0.68 \mu \mathrm{g} \mathrm{g}^{-1}$ of PAs, which might because the Arnebiae Radix was contained in the granules. Even through Asteris Radix Et Rhizoma was also a constituent of anti-acute bronchitis syrup, PAs were not detected in it. This could be due to the elimination of PAs during the drug preparation process. An anti-cervical erosion suppository was also detected with big amount of PAs

Fig. 6 Concentrations of PAs in different commercial herbal drug samples. A, Concentration of single analytes in commercial herbal drug samples; B, total concentration of PAs in commercial herbal drug samples. Test 1 , pharyngitis tablets; test 2, anticough tablets; test 3 , anticough granules 1 ; test 4 , anticough granules 2 ; test 5 , anticough granules for kids; test 6 , slimming tablets; test 7 , anti-cervical erosion suppository; test 8 , anti-acute bronchitis syrup.

( $27.69 \mu \mathrm{g} \mathrm{g}^{-1}$ ), and that would attribute to the extracts of Arnebiae Radix in it. Although the anti-cervical erosion suppository was a kind of drug for external use, the potential toxic effect still existed. Due to the existence of PAs in peppermint, ${ }^{10,22}$ one type of Japanese slimming tablets that contained peppermint was also tested, but no PAs were detected in them. Generally, seven types of commercial herbal drug samples were produced in China, and they were all easily bought in Chinese Pharmacies. Six out of them were detected with PAs. The results indicated that PAs could not be easily eliminated after pharmaceutical processing and they would be ingested by patients or customers.

\section{Conclusions}

In this study, an efficient strategy was established for systematic screening and the determination of PAs in herbal medicines using UPLC-Q-TOF-MS analysis. The sample-preparation procedure was optimized to guarantee the accuracy and sensitivity of this method. On account of the high mass accuracy of TOF-MS and the strong capability of extracting ions of interest accurately, the established method showed excellent merits, such as simplicity, rapidity and accuracy, thereby making the reliable determinations and confirmations of ten PA concentrations in six types of herbal samples possible.

Although people have noticed the existence of PAs and their hepatotoxicity, more or less, Emilia sonchifolia, Asteris Radix Et Rhizoma, Arnebiae Radix, Senecionis Scandentis Hebra, Farfarae Flos, and Senecionis Cannabifolii Herba were still widely used in many countries. There are two possible reasons 
for this phenomenon. Firstly, liver injury cases that resulted from PAs were less reported or less discovered due to the complicated pathogenesis of liver injury and the slow presentation of toxic symptoms induced by PAs. Secondly, most of the researchers mainly considered the pharmacological activity and the efficacy of these herbs, but few systematically studied the contents of PAs in these herbs and the toxicological effects. In this study, we selected ten representative PAs and established the sensitive and accurate UPLC-Q-TOF-MS method to systematically study the type and concentration of PAs in these herbal plant samples and related herbal drug samples. Due to the variation in the genus or in the species, different herbal plant samples were contained with different types and concentrations of PAs, but all of these plant samples had sufficient PAs to show potential toxicity. In addition, six out of eight commercial herbal drug samples contained different types and amounts of PAs. Hence, we suggest to the government regulatory authorities and non-governmental organizations that the use of these six plants as herbal medicine should be limited, or a proper limit inspection of PAs in these herbal plants should be established.

\section{Acknowledgements}

This study was financially supported by the National Natural Science Foundation of China (Nos. 81603073, 81573391, 81503034, and ZSTH-027), Natural Science Foundation of Shaanxi Province (Nos. 2017JQ8026), Fundamental Research Funding of Xi'an Jiaotong University (Nos. xjj2017185 and 1191320026), Postdoctoral Science Foundation of China (No. 2016M602836) and Postdoctoral Science Foundation of Shaanxi Province (No. 2017BSHYDZZ40).

\section{References}

1. J. Qiu, Nature, 2007, 448, 126.

2. E. Roeder, Pharmazie, 2000, 55, 711.

3. J. Jing and R. Teschke, J. Clin. Transl. Hepatol., 2018, 6, 57.

4. R. Teschke and L. Zhang, J. Mol. Pathophysiol., 2015, 4, 85.

5. J. Tang and M. Hattori, Acta Pharmcol. Sin., 2011, 46, 762.

6. Y. Zhu, M. Niu, J. Chen, Z. S. Zou, Z. J. Ma, S. H. Liu, R. L. Wang, T. T. He, H. B. Song, Z. X. Wang, S. B. Pu, X. Ma, L. F. Wang, Z. F. Bai, Y. L. Zhao, Y. G. Li, J. B. Wang, and X. H. Xiao, J. Gastroenterol. Hepatol., 2016, 31, 1476.

7. R. Teschke, L. Zhang, H. Long, A. Schwarzenboeck, W. Schmidt-Taenzer, A. Genthner, A. Wolff, C. Frenzel, J. Schulze, and A. Eickhoff, Ann. Hepatol., 2015, 14, 7.

8. T. Mroczek, K. Glowniak, and A. Wlaszczyk, J. Chromatogr. A, 2002, 949, 249.

9. P. P. Fu, Q. Xia, M. W. Chou, and G. Lin, J. Nanjing Med. Univ., 2005, 19, 1.

10. M. Schulz, J. Meins, S. Diemert, P. Zagermann-Muncke, R. Goebel, D. Schrenk, M. Schubert-Zsilavecz, and M. AbdelTawab, Phytomedicine, 2015, 22, 648.

11. A. R. Mattocks, Nature, 1968, 217, 723.

12. Q. Xia, X. He, L. Ma, S. Chen, and P. P. Fu, Chem. Res. Toxicol., 2018, 31, 619.

13. J. Ma, Q. Xia, P. P. Fu, and G. Lin, J. Food Drug Anal., 2018, 26, 965.

14. K. Betteridge, Y. Cao, and S. M. Colegate, J. Agric. Food Chem., 2005, 53, 1894.

15. "Pharmacopoeia of the People's Republic of China", 2015, The Medicine Science and Technology Press of China,
Beijing, China.

16. D. Tian, Z. Y. Li, J. P. Jia, and X. M. Qin, Anal. Lett., 2014 , 48, 127.

17. U. M. Seo, B. T. Zhao, W. I. Kim, E. K. Seo, J. H. Lee, B. S. Min, B. S. Shin, J. K. Son, and M. H. Woo, Chem. Pharm. Bull., 2015, 63, 546.

18. X. Cheng, M. Liao, X. Diao, Y. Sun, and L. Zhang, Biomed. Chromatogr., 2018, 32, 1.

19. V. M. Couto, F. C. Vilela, D. F. Dias, M. H. Dos Santos, R. Soncini, C. G. Nascimento, and A. Giusti-Paiva, J. Ethnopharmacol., 2011, 134, 348.

20. G. George Kallivalappil and G. Kuttan, Inflammopharmacology, 2019, 27, 409.

21. M. L. Deinzer, B. L. Arbogast, D. R. Buhler, and P. R. Cheeke, Anal. Chem., 2002, 54, 1811.

22. D. Bodi, S. Ronczka, C. Gottschalk, N. Behr, A. Skibba, M. Wagner, M. Lahrssen-Wiederholt, A. Preiss-Weiger, and A. These, Food Addit. Contam., Part A, 2014, 31, 1886.

23. F. Kakar, Z. Akbarian, T. Leslie, M. L. Mustafa, J. Watson, H. P. van Egmond, M. F. Omar, and J. Mofleh, J. Toxicol., 2010, 2010, 1 .

24. A. R. Mattocks, Anal. Chem., 1967, 39, 443.

25. E. M. Mudge, A. M. Jones, and P. N. Brown, Food Addit. Contam., Part A, 2015, 32, 2068.

26. K. A. Beales, K. Betteridge, S. M. Colegate, and J. A. Edgar, J. Agric. Food Chem., 2004, 52, 6664.

27. R. Chizzola, G. Bassler, M. Kriechbaum, and G. Karrer, $J$. Agric. Food Chem., 2015, 63, 1293.

28. S. M. Colegate, S. L. Welsh, D. R. Gardner, J. M. Betz, and K. E. Panter, J. Agric. Food Chem., 2014, 62, 7382.

29. J. F. García-Reyes, M. D. Hernando, A. Molina-Díaz, and A. R. Fernández-Alba, TrAC, Trends Anal. Chem., 2007, 26, 828 .

30. P. Borman and D. Elder, "Q2(R1) Validation of Analytical Procedures", 2017, John Wiley \& Sons, Inc., 127.

31. S. H. Yoon, M. S. Kim, S. H. Kim, H. M. Park, H. Pyo, Y. M. Lee, K. T. Lee, and J. Hong, J. Chromatogr. B, 2015, 992, 56.

32. E. Kowalczyk, Z. Sieradzki, and K. Kwiatek, Food Anal. Methods, 2017, 11, 1345.

33. L. Lorena, M. Roberta, R. Alessandra, M. Clara, and C. Francesca, Food Anal. Methods, 2016, 9, 1825.

34. S. W. C. Chung and C. H. Lam, J. Agric. Food Chem., 2018, 66, 3009.

35. A. C. Valese, L. Molognoni, L. A. D. Ploencio, F. G. de Lima, L. V. Gonzaga, S. L. Gorniak, H. Daguer, F. Barreto, and A. C. O. Costa, Food Control, 2016, 67, 183.

36. Y. Liang, H. Hao, A. Kang, L. Xie, T. Xie, X. Zheng, C. Dai, L. Wan, L. Sheng, and G. Wang, J. Chromatogr. A, 2010, 1217, 4971.

37. I. Ferrer and E. M. Thurman, J. Chromatogr. A, 2007, 1175, 24.

38. F. D. Debelle, J. L. Vanherweghem, and J. L. Nortier, Kidney Int., 2008, 74, 158.

39. F. Willmot and G. Robertson, Lancet, 1920, 196, 848.

40. A. H. Liang and Z. G. Ye, Chin, J. Chin. Mater. Med., 2006 , $31,93$.

41. L. Ahmad, Y. He, J. C. Hao, A. Semotiuk, Q. R. Liu, and P. Mazari, J. Ethnopharmacol., 2018, 210, 88.

42. C. Liu, H. Y. Lu, H. Cheng, and Y. Y. Yu, Infect. Dis. Inform., 2017, 30, 243.

43. J. Tang and M. Cheng, Acta Pharm. Sin., 2019, 54, 420.

44. H. N. Wang and S. Li, Chin. Trad. Herb. Drugs, 2014, 45, 2027.

45. M. Ding, Z. Li, X. A. Yu, D. Zhang, J. Li, H. Wang, J. He, X. M. Gao, and Y. X. Chang, J. Pharm. Biomed. Anal., 2018, 156, 349. 